\title{
Reliability of quantitative sensory testing on myofascial trigger points in the upper trapezius muscle of individuals with chronic neck pain
}

\author{
Almir Vieira Dibai-Filho ${ }^{1}$ (), Alessandra Kelly de Oliveira² ${ }^{(1)}$, Matheus Pereira Oliveira $^{3}$ (), \\ Marília Artese Barros 3 (1), Débora Bevilaqua-Grossi²,3 (), Rinaldo Roberto de Jesus Guirro ${ }^{2,3 *}$ (1)
}

\begin{abstract}
SUMMARY
OBJECTIVE: The objective of this study was to measure the intra- and inter-rater reliability of the quantitative sensory testing for measuring the thermal pain threshold on myofascial trigger points in the upper trapezius muscle of individuals with chronic neck pain.

METHODS: Thirty female participants were included, aged between 18 and 45 years and with bilateral myofascial trigger points, active and centrally located in the upper trapezius muscle. Two measurements with quantitative sensory testing were performed by each examiner at an interval of 1 week between them.

RESULTS: We observed substantial reliability for the intra-rater analysis (intraclass correlation coefficient ranging between 0.876 and 0.896 ) and excellent reliability for the inter-rater analysis (intraclass correlation coefficient ranging between 0.917 and 0.954).

CONCLUSION: The measurement of the thermal pain threshold on myofascial trigger points in individuals with chronic neck pain has acceptable reliability values, supporting the use of the quantitative sensory testing in the research setting and the clinical environment.

KEYWORDS: Myofascial pain syndromes. Reproducibility of results. Pain measurement.
\end{abstract}

\section{INTRODUCTION}

Neck pain is currently the most prevalent musculoskeletal disorders, with an estimated involvement of $50 \%$ of the population ${ }^{1}$. Different anatomical structures may be involved in the pathological process of neck pain, such as ligaments, tendons, nerve roots, and, in particular, the myofascial component ${ }^{2}$.

Studies show that individuals with musculoskeletal disorders have vascular, metabolic, electromyographic, and thermographic changes ${ }^{3,4}$. In addition, a common clinical sign in patients with neck pain is the presence of myofascial trigger points, especially in the upper trapezius muscle ${ }^{3}$.

Regarding the assessment of myofascial pain, Simons et al..$^{5}$ presented the method of diagnosing the myofascial trigger points centered on palpation and, in general, this is the most accepted method both in research studies and the clinical practice. However, due to the complexity existing in the evaluation of the painful experience, other methods have been used to complement such assessment, such as algometry, thermography ${ }^{3}$, and skin impedance ${ }^{6}$.

Within this context, quantitative sensory testing (QST) is another plausible tool to be used in the presence of myofascial trigger points, since it involves a set of methods to assess somatosensory function, including measuring the presence of hyperalgesia and allodynia ${ }^{7}$. It is noteworthy that the myofascial trigger points actively participate in the peripheral and central sensitization processes, as highlighted by important studies ${ }^{8-10}$.

Nevertheless, despite the evaluative potential of the QST in patients with myofascial trigger points, for the correct clinical use of this tool, it is necessary to identify the amount of error inherent to the use of the QST in this population. Thus, the aim of this study was to evaluate the intra- and inter-rater reliability of the QST in measuring thermal pain thresholds on myofascial trigger points in the upper trapezius muscle in patients with chronic neck pain. The hypothesis of this study is that the QST has adequate reliability.

\footnotetext{
${ }^{1}$ Universidade Federal do Maranhão, Postgraduate Program in Physical Education - São Luís (MA), Brazil.

${ }^{2}$ Universidade de São Paulo, Ribeirão Preto Medical School, Department of Health Sciences, Postgraduate Program in Rehabilitation and Functional Performance - Ribeirão Preto (SP), Brazil.

${ }^{3}$ Universidade de São Paulo, Ribeirão Preto Medical School, Department of Health Sciences, Physical Therapy Course - Ribeirão Preto (SP), Brazil. *Corresponding author: rguirro@fmrp.usp.br

Conflicts of interest: the authors declare there is no conflicts of interest. Funding: Fundação de Amparo à Pesquisa do Estado de São Paulo (FAPESP, grants 2013/19368-8 and 2013/09753-1), Coordenação de Aperfeiçoamento de Pessoal de Nível Superior (CAPES, Finance Code 001) and Conselho Nacional de Desenvolvimento Científico e Tecnológico (PIBIC-CNPq).
}

Received on September 14, 2021. Accepted on September 16, 2021. 


\section{METHODS}

\section{Ethical aspects}

The project was approved by the Research Ethics Committee of the institution (opinion number 030643/2013), and the data collection was carried out at the Physiotherapeutic Resources Laboratory. The recruitment of volunteers took place in the communities near the university through verbal dissemination, posters, and social media. Once selected, the volunteers were instructed about all the study procedures, objectives, and characteristics, and validated their participation by signing the free and informed consent form.

\section{Study design}

This is a reliability study of the QST instrument for measuring the painful response to heat and cold stimuli on myofascial trigger points in women with chronic neck pain, considering different times and different examiners. The researchers responsible for performing the examination were unaware of the participants' pain characteristics (i.e., pain intensity, pressure pain threshold, chronicity, and disability).

\section{Sample}

The sample calculation considered a confidence coefficient of 0.95 and confidence interval amplitude for the intraclass correlation coefficient (ICC) of 0.30 . In addition, the calculation was performed to detect moderate reliability $(\mathrm{ICC}=0.75$ ) according to the study conducted by Fleiss ${ }^{11}$. Thus, a minimum sample size of 24 participants was estimated. The processing of the sample calculation was performed based on the study conducted by Bonett ${ }^{12}$.

As diagnostic criteria for chronic neck pain, a score of $\geq 5$ on the Neck Disability Index (NDI), a score of $\geq 3$ on the Numeric Pain Rating Scale (NPRS) at rest or during active cervical movement, and the presence of pain for more than 3 months were considered. In addition, the volunteers had bilateral and active myofascial trigger points in the upper trapezius muscle identified according to the diagnostic criteria established by Simons et al. ${ }^{5}$ and Gerwin et al. ${ }^{13}$, as follows: presence of a tense band in the upper trapezius muscle; presence of a hypersensitive point within the tight band; local twitch in response to palpation of the tight band; and reproduction of referred pain due to compression of $2.5 \mathrm{~kg} / \mathrm{cm}^{2}$ on the trigger point.

The myofascial trigger point was considered active when the participant presented spontaneous pain or reported a familiar pain while performing the compression ${ }^{5}$. Diagnostic criteria were applied by a physical therapist with 8 years of experience in myofascial pain.
Exclusion criteria were: history of cervical trauma; head, face, or cervical surgery; cervical hernia; degenerative spinal diseases; having undergone physical therapy treatment for neck pain in the last 3 months; use of analgesics, anti-inflammatory drugs, or muscle relaxants in the last week; and presence of systemic or autonomic diseases or diagnosis of fibromyalgia.

\section{Assessment procedures}

The assessment procedures were carried out as follows: a researcher with experience in measuring the painful experience applied the pain assessment instruments and identified the presence of myofascial trigger points at an initial moment; in a second moment, two other examiners previously trained and familiarized with the use of the QST carried out the evaluations of the thermal pain threshold in two moments at an interval of 1 week between them ${ }^{14}$.

Data to fit the participants in the eligibility criteria were initially collected. The NPRS was used to assess pain intensity ${ }^{15}$, the NDI was used to assess the neck disability in the presence of pain ${ }^{16}$, and the pressure pain threshold assessment was performed using a digital algometer model PTR-300 (Instrutherm, São Paulo, Brazil $)^{17}$.

\section{Quantitative sensory testing}

The evaluation of the thermal pain threshold was performed using the QST (TSA II Neurosensory Analyzer, Medoc, Ramat Yishai, Israel). The environmental evaluation remained at a controlled temperature of $23^{\circ} \mathrm{C}$. For collection, the participant maintained the sitting position and the examiner positioned the equipment electrode over the myofascial trigger points in the upper trapezius muscle. The order of the side to be evaluated was defined by drawing lots before each evaluation.

For collection, three repetitions of the test were performed for each stimulus (hot or cold): the thermal pain threshold with heat had an initial temperature of $32^{\circ} \mathrm{C}$ and a maximum of $50^{\circ} \mathrm{C}$, while the cold had an initial temperature of $32^{\circ} \mathrm{C}$ and a minimum of $0^{\circ} \mathrm{C}$.

The volunteer was initially familiarized with the instrument: a test was performed in the palm region of the hand. During the examination on the trigger point, the volunteer was instructed to interrupt the procedure by pressing a switch whenever the temperature caused her pain, and the temperature value was then recorded. For statistical analysis, the mean of the three repetitions was used.

\section{Statistical analysis}

This study was carried out based on the Guidelines for Reporting Reliability and Agreement Studies (GRRAS) ${ }^{18}$, and the ICC was 
used to determine the intra- and inter-rater reliability of the thermal pain threshold, with its respective $95 \%$ confidence interval, standard error of measurement, and minimum detectable difference (MDD). The interpretation of the ICC value was based on the Fleiss study: low reliability (ICC<0.40), moderate (ICC between 0.40 and 0.75 ), substantial (ICC between 0.75 and 0.90 ), and excellent $(\text { ICC }>0.90)^{11}$. Data processing was performed in the Statistical Package for the Social Sciences, version 17.0 (Chicago, IL, USA).

\section{RESULTS}

Forty volunteers were recruited, but 11 were excluded for not reaching the inclusion criteria. The final sample consisted of 29 women, who were right-handed with a mean age of 22.03 years [standard deviation $(\mathrm{SD})=3.66$ ] and a mean body mass index of $23.52 \mathrm{~kg} / \mathrm{m}^{2}(\mathrm{SD}=3.55)$.
Mean pain intensity was 3.07 points $(S D=1.57)$ at rest and 4.97 points $(S D=3.69)$ after active cervical movement, with mean pain chronicity of 41.00 months $(S D=32.36)$. Mean disability was $10.07(\mathrm{SD}=3.81)$. The pressure pain thresholds on the left and right myofascial trigger points were $1.77 \mathrm{~kg} / \mathrm{cm}^{2}(\mathrm{SD}=0.44)$ and $1.78 \mathrm{~kg} / \mathrm{cm}^{2}(\mathrm{SD}=0.52)$, respectively. The thermal pain threshold values of the two evaluators are given in Table 1.

Table 2 presents the intra-rater reliability values of the QST measurement. Substantial reliability was observed, with ICC values between 0.876 and 0.896 , SEM between 1.03 and $3.38^{\circ} \mathrm{C}$, and $\mathrm{MDD}$ between 2.85 and $8.99^{\circ} \mathrm{C}$.

The inter-rater reliability values demonstrated excellent reliability, with ICC values between 0.917 and 0.954, SEM between 0.68 and $2.17^{\circ} \mathrm{C}$, and MDD between 1.88 and $6.01^{\circ} \mathrm{C}$ (Table 3).

Table 1. Values of the thermal pain threshold $\left({ }^{\circ} \mathrm{C}\right)$ evaluated using quantitative sensory testing according to the measurements of the two evaluators $(n=29)$.

\begin{tabular}{l|c|c|c|c}
\multirow{2}{*}{ QST } & \multicolumn{2}{|c|}{ Examiner 1 } & \multicolumn{2}{c}{ Examiner 2 } \\
\cline { 2 - 5 } & Test & Retest & Test & Retest \\
\hline RUT heat & $44.40(2.99)$ & $45.53(2.89)$ & $44.29(2.96)$ & $44.62(3.13)$ \\
\hline RUT cold & $18.72(9.47)$ & $15.39(10.07)$ & $18.07(8.86)$ & $17.01(9.57)$ \\
\hline LUT heat & $44.07(3.18)$ & $45.38(3.19)$ & $43.69(2.89)$ & $44.45(3.02)$ \\
\hline LUT cold & $19.57(8.89)$ & $15.88(10.68)$ & $17.49(9.27)$ & $17.02(8.78)$ \\
\hline
\end{tabular}

Values are shown as mean (standard deviation).

RUT: right upper trapezius; LUT: left upper trapezius.

Table 2. Intra-rater reliability of the measurement of the thermal pain threshold in patients with chronic neck pain ( $n=29)$.

\begin{tabular}{l|c|c|c|c|c|c}
\hline QST & ICC & $95 \%$ CI & SEM $\left({ }^{\circ} \mathbf{C}\right)$ & SEM (\%) & MDD ( $\left.{ }^{\circ} \mathbf{C}\right)$ & MDD (\%) \\
\hline RUT heat & 0.876 & $0.740,0.941$ & 1.04 & 2.30 & 2.87 & 6.38 \\
\hline RUT cold & 0.879 & $0.745,0.942$ & 3.38 & 18.50 & 8.99 & 51.28 \\
\hline LUT heat & 0.896 & $0.781,0.950$ & 1.03 & 2.30 & 2.85 & 6.37 \\
\hline LUT cold & 0.895 & $0.779,0.950$ & 2.91 & 16.87 & 8.07 & 46.75 \\
\hline
\end{tabular}

QST: quantitative sensory testing; RUT: right upper trapezius; LUT: left upper trapezius; ICC: intraclass correlation coefficient; CI: confidence interval; SEM: standard error of measurement; MDD: minimum detectable difference.

Table 3. Inter-rater reliability of the measurement of the thermal pain threshold in patients with chronic neck pain ( $n=29)$.

\begin{tabular}{l|c|c|c|c|c|c}
\hline QST & ICC & $95 \%$ CI & SEM $\left({ }^{\circ} \mathbf{C}\right)$ & SEM (\%) & MDD ( $\left.{ }^{\circ} \mathbf{C}\right)$ & MDD (\%) \\
\hline RUT heat & 0.948 & $0.892,0.975$ & 0.68 & 1.53 & 1.88 & 4.24 \\
\hline RUT cold & 0.954 & $0.904,0.978$ & 2.09 & 11.36 & 5.79 & 31.49 \\
\hline LUT heat & 0.917 & $0.825,0.960$ & 0.87 & 1.99 & 2.42 & 5.52 \\
\hline LUT cold & 0.943 & $0.881,0.973$ & 2.17 & 11.70 & 6.01 & 32.43 \\
\hline
\end{tabular}

QST: quantitative sensory testing; RUT: right upper trapezius; LUT: left upper trapezius; ICC: intraclass correlation coefficient; Cl: confidence interval; SEM: standard error of measurement; MDD: minimum detectable difference. 


\section{DISCUSSION}

The present study showed adequate reliability in the thermal pain threshold on myofascial trigger points in the upper trapezius muscle while considering different times and different examiners. The evaluation of the pain threshold using a heat stimulus showed a smaller amount of error than the evaluation of the pain threshold by the cold stimulus due to the greater variability than the perception of pain with cold. Thus, the thermal pain threshold can be used in the clinical context to assess the somatosensory system as an outcome measure of clinical interventions.

Clinical research on pain is constantly growing due to the emergence of new assessment tools and methods and, in this context, the QST is widely used for the assessment of skin sensation and the sensitive assessment of deep tissues, such as muscles, fascia, ligaments, and viscera ${ }^{19}$. In addition, the QST is able to inform about the functionality of the somatosensory system, quantifying the presence and intensity of sensory phenomena (such as loss or gain of function, hyperalgesia or hypoalgesia, and allodynia), thus contributing to the assessment of various painful conditions ${ }^{20,21}$.

With an increase in the clinical use of the QST in different conditions, there is a need for studies to ensure the reliability of this instrument in each specific clinical condition. A systematic review investigating the reliability of thermal QST observed that in 21 studies included, only 5 had high methodological quality. In addition, most studies have been done in healthy patients and in diseases that involve the nervous system, such as neuropathies ${ }^{22}$. The present study was carried out with methodological rigor based on the GRRAS ${ }^{18}$. Another important point of our study is to verify the reliability in a sample not reported yet in published studies, i.e., myofascial pain.

Considering the reliability of the QST in other painful conditions, some studies have investigated orofacial pain ${ }^{23}$, knee osteoarthritis ${ }^{24}$, and musculoskeletal traumatic injury ${ }^{25}$. Our results found the ICC values similar to the ones in the aforementioned studies, indicating a pattern of error in the measurements performed with the QST, regardless of the population with pain studied.

\section{REFERENCES}

1. Hogg-Johnson S, van der Velde G, Carroll LJ, Holm LW, Cassidy JD, Guzman J, et al. The burden and determinants of neck pain in the general population: results of the Bone and Joint Decade 2000-2010 Task Force on Neck Pain and Its Associated Disorders. Spine (Phila Pa 1976). 2008;33(4 Suppl.):S39-51. https://doi. org/10.1097/BRS.0b013e31816454c8
Pigg et al. ${ }^{23}$ evaluated the reliability of the QST to verify the somatosensory function in patients with pain related to the trigeminal nerve. The measurements were made on the skin of the right cheek, the tip of the tongue, and bilaterally on the gingival mucosa of the upper premolar region, and the authors found the ICC values ranging between 0.41 and 0.89 for inter-rater reliability and between 0.43 and 0.87 for intra-rate reliability. Wylde et al. ${ }^{24}$ evaluated the test-retest reliability of the thermal pain threshold in patients with knee osteoarthritis and found the ICC values ranging from 0.59 to 0.83 .

Middlebrook et al..$^{25}$ measured the inter-rater reliability of the QST in the assessment of individuals with traumatic musculoskeletal injury and found the ICC values ranging from 0.57 to 0.94 . Our study identified less variation of ICC in the measurements of the thermal pain threshold; however, our sample consisted of patients with chronic pain ( $>3$ months of pain).

The study has limitations that must be considered. Our study included only women due to the higher prevalence of myofascial pain in this gender. In addition, menstrual periods and contraceptive use were not controlled. This is an important limitation since the literature shows variations in the sensation of pain in different phases of the menstrual cycle $^{26}$.

\section{CONCLUSION}

The measurement of the thermal pain threshold on myofascial trigger points in individuals with chronic neck pain presents acceptable reliability values while considering different times and examiners, which supports the use of this method of assessment for data collection in research and the clinical environment.

\section{AUTHORS' CONTRIBUTIONS}

AVDF, RRJG: Conceptualization, Data curation, Formal Analysis, Methodology, Project administration, Writing - review \& editing. AKO, MPO, MAB: Conceptualization, Data curation, Formal Analysis, Methodology, Writing - original draft. DBG: Conceptualization, Methodology, Writing - review \& editing. 
4. Larsson R, Öberg PÅ, Larsson S-E. Changes of trapezius muscle blood flow and electromyography in chronic neck pain due to trapezius myalgia. Pain. 1999;79(1):45-50. https://doi.org/10.1016/ S0304-3959(98)00144-4

5. Simons DG, Simons LS, Travell JG. Travell \& Simons' myofascial pain and dysfunction: the trigger point manual. 2nd ed. LWW; 1998. Available from: https://www.amazon.com.br/Travell-SimonsMyofascial-Pain-Dysfunction/dp/0683083635 [Accessed 24th October 2020].

6. Dibai-Filho AV, Barros MA, Oliveira AK, Jesus Guirro RR. Electrical impedance of the torso is associated with the pressure pain threshold on myofascial trigger points in patients with chronic neck pain: a cross-sectional study. J Back Musculoskelet Rehabil. 2018;31(2):275-84. https://doi.org/10.3233/BMR-169671

7. Hendriks E, Voogt L, Lenoir D, Coppieters I, Ickmans K. Convergent validity of the central sensitization inventory in chronic whiplashassociated disorders; associations with quantitative sensory testing, pain intensity, fatigue, and psychosocial factors. Pain Med. 2020;21(12):3401-12. https://doi.org/10.1093/pm/pnaa276

8. XuY-M, GeH-Y,Arendt-Nielsen L. Sustained nociceptive mechanical stimulation of latent myofascial trigger point induces central sensitization in healthy subjects. J Pain. 2010;11(12):1348-55. https://doi.org/10.1016/j.jpain.2010.03.010

9. Ge H-Y, Arendt-Nielsen L. Latent myofascial trigger points. Curr Pain Headache Rep. 2011;15(5):386-92. https://doi.org/10.1007/ s11916-011-0210-6

10. Fernández-Lao C, Cantarero-Villanueva I, Fernández-De-LasPeñas C, Del-Moral-Ávila R, Arendt-Nielsen L, Arroyo-Morales M. Myofascial trigger points in neck and shoulder muscles and widespread pressure pain hypersensitivtiy in patients with postmastectomy pain: evidence of peripheral and central sensitization. Clin J Pain. 2010;26(9):798-806. https://doi. org/10.1097/AJP.0b013e3181f18c36

11. Fleiss JL. The design and analysis of clinical experiments. Wiley; 1999.

12. Bonett DG. Sample size requirements for estimating intraclass correlations with desired precision. Stat Med. 2002;21(9):1331-5. https://doi.org/10.1002/sim.1108

13. Gerwin RD, Shannon S, Hong CZ, Hubbard D, Gevirtz R. Interrater reliability in myofascial trigger point examination. Pain. 1997;69(12):65-73. https://doi.org/10.1016/S0304-3959(96)03248-4

14. Dibai-Filho AV, Guirro ECO, Ferreira VTK, Brandino HE, Vaz MMOLL, Jesus Guirro RR. Reliability of different methodologies of infrared image analysis of myofascial trigger points in the upper trapezius muscle. Braz J Phys Ther. 2015;19(2):122-8. https:// doi.org/10.1590/bjpt-rbf.2014.0076
15. Ferreira-Valente MA, Pais-Ribeiro JL, Jensen MP. Validity of four pain intensity rating scales. Pain. 2011;152(10):2399-404. https:// doi.org/10.1016/j.pain.2011.07.005

16. Cook C, Richardson JK, Braga L, Menezes A, Soler X, Kume P, et al. Cross-cultural adaptation and validation of the Brazilian Portuguese version of the neck disability index and neck pain and disability scale. Spine (Phila Pa 1976). 2006;31(14):1621-7. https://doi.org/10.1097/01.brs.0000221989.53069.16

17. Nussbaum EL, Downes L. Reliability of clinical pressure-pain algometric measurements obtained on consecutive days. Phys Ther. 1998;78(2):160-9. https://doi.org/10.1093/ptj/78.2.160

18. Kottner J,Audigé L, Brorson S, Donner A, Gajewski BJ, Hróbjartsson A, et al. Guidelines for Reporting Reliability and Agreement Studies (GRRAS) were proposed. J Clin Epidemiol. 2011;64(1):96-106. https://doi.org/10.1016/j.jclinepi.2010.03.002

19. Arendt-Nielsen L, Yarnitsky D. Experimental and clinical applications of quantitative sensory testing applied to skin, muscles and viscera. J Pain. 2009;10(6):556-72. https://doi.org/10.1016/j. jpain.2009.02.002

20. Rowbotham MC, Fields HL. The relationship of pain, allodynia and thermal sensation in post-herpetic neuralgia. Brain. 1996;119(2):347-54. https://doi.org/10.1093/brain/119.2.347

21. Maier C, Baron R, Tölle TR, Binder A, Birbaumer N, Birklein F, et al. Quantitative sensory testing in the German Research Network on Neuropathic Pain (DFNS): somatosensory abnormalities in 1236 patients with different neuropathic pain syndromes. Pain. 2010;150(3):439-50. https://doi.org/10.1016/j.pain.2010.05.002

22. Moloney NA, Hall TM, Doody CM. Reliability of thermal quantitative sensory testing: a systematic review. J Rehabil Res Dev. 2012;49(2):191-208. https://doi.org/10.1682/JRRD.2011.03.0044

23. Pigg M, Baad-Hansen L, Svensson P, Drangsholt M, List T. Reliability of intraoral quantitative sensory testing (QST). Pain. 2010;148(2):220-6. https://doi.org/10.1016/j.pain.2009.10.024

24. Wylde V, Palmer S, Learmonth ID, Dieppe P. Test-retest reliability of quantitative sensory testing in knee osteoarthritis and healthy participants. Osteoarthr Cartil. 2011;19(6):655-8. https://doi. org/10.1016/j.joca.2011.02.009

25. Middlebrook N, Heneghan NR, Evans DW, Rushton A, Falla D. Reliability of temporal summation, thermal and pressure pain thresholds in a healthy cohort and musculoskeletal trauma population. PLoS One. 2020;15(5):e0233521. https://doi. org/10.1371/journal.pone.0233521

26. Piroli A, Mattei A, Carta G, D'Alfonso A, Palermo P, Marinangeli F, et al. Influence of the menstrual cycle phase on pain perception and analgesic requirements in young women undergoing gynecological laparoscopy. Pain Pract. 2019;19(2):140-8. https:// doi.org/10.1111/PAPR.12727 